\title{
Renewable Powered Cellular Networks: Energy Field Modeling and Network Coverage
}

\author{
Kaibin Huang, Marios Kountouris and Victor O. K. Li
}

\begin{abstract}
Powering radio access networks using renewables, such as wind and solar power, promises dramatic reduction in the network operation cost and the network carbon footprints. However, the spatial variation of the energy field can lead to fluctuations in power supplied to the network and thereby affects its coverage. This warrants research on quantifying the aforementioned negative effect and designing countermeasure techniques, motivating the current work. First, a novel energy field model is presented, in which fixed maximum energy intensity $\gamma$ occurs at Poisson distributed locations, called energy centers. The intensities fall off from the centers following an exponential decay function of squared distance and the energy intensity at an arbitrary location is given by the decayed intensity from the nearest energy center. The product between the energy center density and the exponential rate of the decay function, denoted as $\psi$, is shown to determine the energy field distribution. Next, the paper considers a cellular downlink network powered by harvesting energy from the energy field and analyzes its network coverage. For the case of harvesters deployed at the same sites as base stations (BSs), as $\gamma$ increases, the mobile outage probability is shown to scale as $\left(c \gamma^{-\pi \psi}+p\right)$, where $p$ is the outage probability corresponding to a flat energy field and $c$ is a constant. Subsequently, a simple scheme is proposed for counteracting the energy randomness by spatial averaging. Specifically, distributed harvesters are deployed in clusters and the generated energy from the same cluster is aggregated and then redistributed to BSs. As the cluster size increases, the power supplied to each BS is shown to converge to a constant proportional to the number of harvesters per BS. Several additional issues are investigated in this paper, including regulation of the power transmission loss in energy aggregation and extensions of the energy field model.
\end{abstract}

Index Terms-Cellular networks, renewable energy sources, energy harvesting, stochastic processes.

\section{INTRODUCTION}

The exponential growth of mobile data traffic causes the energy consumption of radio access networks, such as cellular and WiFi networks, to increase rapidly. This not only places heavy burdens on both the electric grid and the environment, but also leads to huge network operation cost. A promising solution for energy conservation is to power the networks using alternative energy sources, which will be a feature of future green telecommunication networks [1]. However, the spatial randomness of renewable energy can severely degrade the performance of large-scale networks, hence it is a fundamental issue to address in network design. Considering a cellular network with renewable powered base stations (BSs),

K. Huang and V. O. K. Li are with the Dept. of Electrical and Electronic Engineering at The University of Hong Kong, Hong Kong. Email: huangkb@eee.hku.hk, vli@eee.hku.hk. M. Kountouris is with the Mathematical and Algorithmic Sciences Lab, Huawei France R\&D, Paris, France. Email: marios.kountouris@ huawei.com. Part of this work has been presented at IEEE Intl. Conf. on Comm. Systems (ICCS) in Nov. 2014. this paper addresses the aforementioned issue by proposing a novel model of the energy field and quantifying the relation between its parameters and network coverage. Furthermore, the proposed technique of energy aggregation is shown to effectively counteract energy spatial randomness.

Studying large-scale energy harvesting networks provides useful insight to network planning and architecture design. This has motivated researchers to investigate the effects of both the spatial and temporal randomness of renewables on the coverage of different wireless networks spread over the horizontal plane [2]-[5]. Poisson point processes (PPPs) are used to model transmitters of a mobile ad hoc network (MANET) in [2] and BSs of a heterogeneous cellular network [3]. Energy arrival processes at different transmitters are usually modeled as independent and identically distributed (i.i.d.) stochastic processes, reducing the effect of energy temporal randomness to independent on/off probabilities of transmitters. Thereby, under an outage constraint, the conditions on the network parameters, such as transmission power and node density of the MANET [2] and densities of different tiers of BSs [3], can be analyzed for a given distribution of energy arrival processes. The assumption of spatially independent energy distributions is reasonable for specific types of renewables that can power small devices, such as kinetic energy and electromagnetic (EM) radiation, but does not hold for primary sources, namely wind and solar power. To some extent, energy spatial correlation is accounted for in [4], [5] but limited to EM radiation. It is proposed in [4] that nodes in a cognitive-radio network opportunistically harvest energy from radiations from a primary network, besides intelligent sharing of its spectrum. The idea of deploying dedicated stations for supplying power wirelessly to energy harvesting mobiles in a cellular network is explored in [5]. In [4], [5], radiations by transmitters with reliable power supply form an EM energy field and its spatial correlation is determined by the EM wave propagation that does not apply to other types of renewables such as wind and solar power.

It is worth mentioning that the analysis and design of large-scale wireless networks using stochastic geometry and geometric random graphs [6]-[8] has been a key research area in wireless networking in the past decade. Similar mathematical tools have also been widely used in the area of geostatistics concerning spatial statistics of natural resources including renewables, where research focuses on topics such as model fitting, estimation, and prediction of energy fields [9], [10]. The two areas naturally merge in the new area of large-scale wireless networks with energy harvesting. It is in this largely uncharted area that the current work makes some 


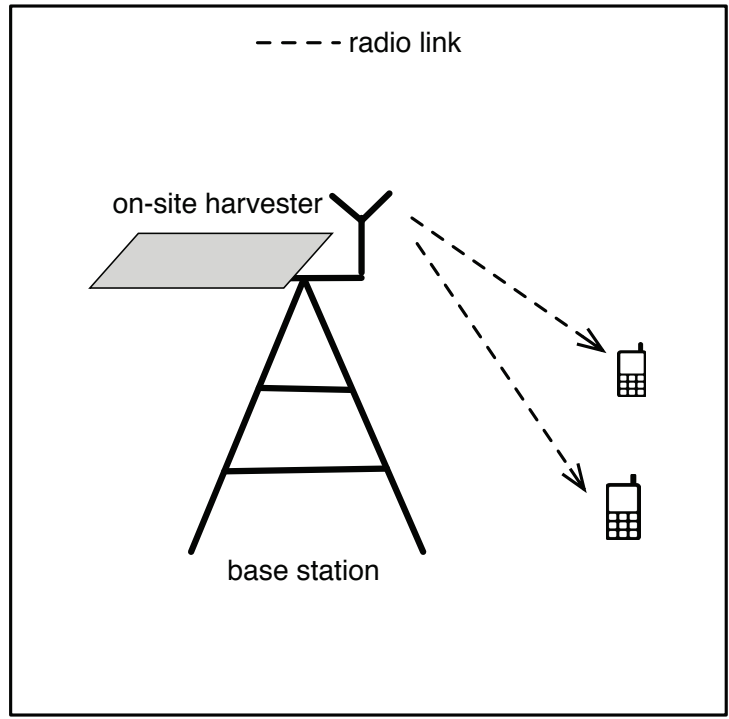

(a) Base station powered by an on-site harvester.

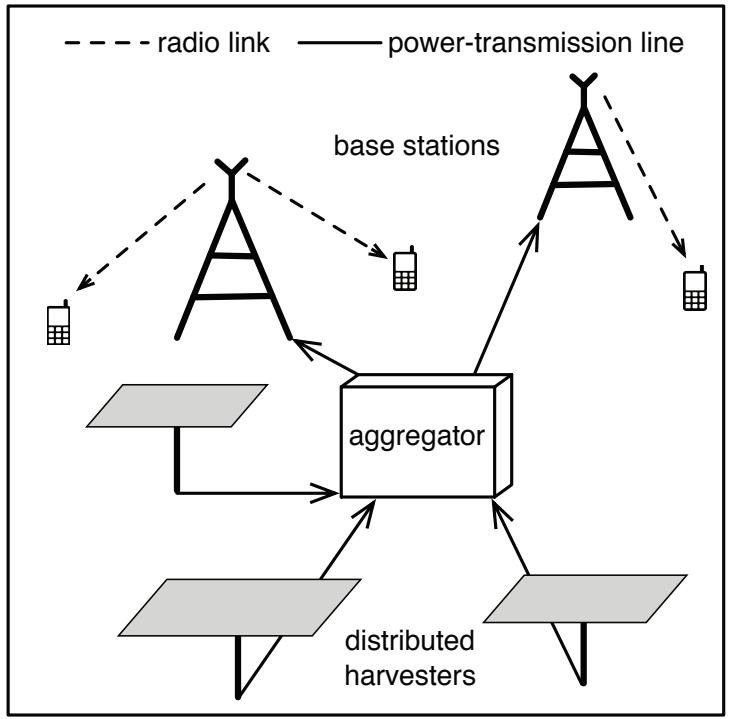

(b) Base stations powered by distributed harvesters.

Fig. 1. Base stations are powered by energy harvesting using either (a) on-site harvesters or (b) distributed harvesters.

initial contributions.

Intermittence of renewables introduces stochastic constraints on available transmission power for a wireless device. This calls for revamping classic information and communication theories to account for such constraints and it has recently attracted extensive research efforts [11]-[17]. As shown in [11] from an information-theoretic perspective, it is possible to avoid capacity loss for an AWGN channel due to energy harvesting provided that a battery with infinite capacity is deployed to counteract the energy randomness. From the communication-theoretic perspective, optimal power control algorithms are proposed in [12], [13] for single-user systems with energy harvesting, which adapt to the energy arrival profile and channel state so as to maximize the system throughput. These approaches have been extended to design more complex energy harvesting systems, such as interference channels [14] and relay channels [15], to design medium access protocols [16], and to account for practical factors such as non-ideal batteries [17]. The prior work assumes fast varying renewable energy sources such as kinetic activities and EM radiation for which adaptive transmission proves an effective way for coping with energy temporal randomness. In contrast, alternative sources targeted in this paper, namely wind and solar radiation, may remain static for minutes to hours, which are orders of magnitude longer than the typical packet length of milliseconds. In other words, these sources are characterized by a high level of spatial randomness but very gradual temporal variations. Thus, this paper focuses on counteracting energy spatial randomness instead of adaptive transmission that is ineffective for this purpose.

In practice, spatial renewable energy maps are created by interpolating data collected from sparse measurement stations separated by distances typically of tens to hundreds of kilometers [18]-[20]. The measurement data is too coarse for constructing the energy field targeting next-generation small cell networks with cell radiuses as small as tens of meters. Furthermore, the field depends not only on atmospherical conditions (e.g., cloud formation and mobility) but also on the harvester/BS deployment environment such as locations and heights of buildings, trees and cell sites. Mapping the energy field requires complex datasets that are difficult to obtain. Even if such datasets are available, the resultant energy-field model may not allow tractable network performance analysis. This motivates the current approach of modeling energy fields using spatial random processes derived from PPPs. Such processes with a well developed theory are widely agreed to be suitable models for natural phenomena and resources [9], [21] as well as random wireless networks [6] and thus provide a desirable tradeoff between tractability and practicality. Note that stochastic-geometry network models in the literature were developed to overcome the same difficulty of lacking real data [6]. In the proposed model, the random locations of fixed maximum energy intensity (denoted as $\gamma$ ), called energy centers, are modeled as a PPP with density $\lambda_{e}$, corresponding to sites on top of tall buildings with exposure to direct sunshine or strong winds. From an energy center, the energy intensity decays exponentially with the squared distance normalized by a constant $\nu$, called the shape parameter, specifying the area of influence by the said center. It is worth mentioning that the decay function is popularly used in solar-field mapping [22] and atmospheric mapping [23]. The energy intensity at an arbitrary location is then given by the decayed intensity with respect to the nearest energy center. The characteristic parameter $\psi$ of the energy field, defined as $\psi=\lambda_{e} \nu$, is shown later to determine its distribution. It is worth mentioning that general insights obtained in this work using the above model, such as that increasing energy spatial correlation reduces outage probability, also hold for other models so long as they 
observe the basic principle of renewable energy field: energy spatial correlation between two locations increases as their separation distance decreases and vice versa [19].

In the paper, BSs of the cellular network are assumed to be deployed on a hexagonal lattice while mobiles are distributed as a PPP. The network is assumed to operate in the noise-limited regime where interference is suppressed using techniques such as orthogonal multiple access or multi-cell cooperation. The regime is most interesting from the energy harvesting perspective since network performance is sensitive to variations of transmission powers or equivalently, harvested energy. For instance, it has been shown that in the interference limited regime, the network coverage is independent of BS transmission power since it varnishes in the expression for the signal-to-interference-and-noise ratio with noise removed [24]. A mobile is said to be under (network) coverage if an outage constraint is satisfied and the outage probability is the performance metric. Each BS allocates transmission power simultaneously to mobiles, either by equal division of the available power, called channel-independent transmission, or by channel inversion, called channel inversion transmission. As illustrated in Fig. 1, each BS is powered by either an onsite harvester or a remote (energy) aggregator that collects energy generated by a cluster of nearby distributed harvesters over transmission lines. Aggregators and distributed harvesters are deployed on hexagonal lattices with densities $\lambda_{a}$ and $\lambda_{h}$, respectively. Connecting harvesters to their nearest aggregators form harvester clusters. Note that energy aggregation is based on the same principle of tackling energy randomness by energy sharing as other existing techniques designed for renewable powered cellular systems (see e.g., [25], [26]). Prior work focuses on algorithmic design while the current analysis targets network performance.

The main contribution of this work is the establishment of a new approach for designing large-scale renewable powered wireless networks that models the energy field using stochastic geometry and applies such a model to network design and performance analysis. The analysis based on the approach allows the interplay of different mathematical tools such as stochastic geometry theory, probabilistic inequalities and large deviation theory, leading to the following new findings.

- Consider the on-site harvester case. If the characteristic parameter $\psi$ is small $(\psi \leq 1 / \pi)$ and the maximum energy density $\gamma$ is large $(\gamma \rightarrow \infty)$, the outage probability monotonically decreases with increasing $\psi$ and $\gamma$ in the form of $\left(c \gamma^{-\pi \psi}+p\right)$ with $p$ being the probability corresponding to a flat energy field and $c$ a constant. The result holds for both channel inversion and channelindependent transmissions. Despite this similarity, the former outperforms the latter by adapting transmission power to mobiles' channels.

- Next, consider distributed harvesters and define the size of a harvester cluster as $\lambda_{h} / \lambda_{a}$. As the cluster size increases, energy aggregation is shown to counteract the spatial randomness of the energy field and thereby stabilize the power supply for BSs. Specifically, the power it distributes to each BS converges to a constant proportional to the number of harvesters per BS. In other words, the energy field becomes a reliable power supply for the network.

- However, an insufficiently high voltage used by harvesters for power transmission to aggregators can incur significant energy loss. It is found that the loss can be regulated by increasing the voltage inversely with the aggregator density to the power of $\frac{1}{4}$.

- Last, key results are extended to two variations of the energy field model characterized by a shot noise process and a power-law energy decay function, respectively.

The remainder of the paper is organized as follows. The mathematical models and metrics are described in Section II. The energy field model is proposed and its properties characterized in Section III. The network coverage is analyzed for the cases of on-site and distributed harvesters in Sections IV and $\mathrm{V}$, respectively. The extensions of the energy field model are studied in Section VI. Simulation results are presented in Section VII followed by concluding remarks in Section VIII.

\section{Models And Metrics}

The spatial models for the energy field, energy harvesters, and cellular network are described in the subsections. The notations are summarized in Table I.

\section{A. Energy Harvester Model}

Aggregation loss arises in the scenario of distributed harvesters, corresponding to the power loss due to transmissions from harvesters to aggregators over cables (see Fig. 2). It is impractical to assume high voltage transmission from distributed harvesters having small form factors and thus aggregation loss can be significant, which is analyzed in the sequel. On the other hand, each aggregator supplies power to $\lambda_{b} / \lambda_{a}$ BSs also over cables, assuming that $\lambda_{b} / \lambda_{a}$ is an integer for simplicity. Aggregators are assumed to be much larger than harvesters and thus can afford having relatively large transformers. Thus, high voltages can be reasonably assumed for power distribution such that the power loss is negligible. Consequently, the specific graph of connections between aggregators and BSs has no effect on the analysis except for the number of BSs each aggregator supports.

The energy field is represented by $\Psi$, which is determined by the function $g(X)$ mapping a location $X \in \mathbb{R}^{2}$ to energy intensity. The discussion of the stochastic geometry model of the energy field is postponed to Section III, whereas its relation with energy harvesting is described below. Let $g(X, t)$ represent the time-varying version of $g(X)$ with $t$ denoting time. Harvesters are assumed to be homogeneous and time is partitioned into slots of unit duration. Let $\eta \in(0,1)$ denote a constant combining factors such as the harvester physical configuration and conversion efficiency, referred to as the harvester aperture by analogy with the antenna aperture. Then the amount of energy harvested at $X$ in the $n$-th slot is $\eta g_{n}(X)$ or equivalently $\eta \int_{n}^{n+1} g(X, t) d t$.

\section{B. Cellular Network Model}

As illustrated in Fig. 2, the traditional model of a cellular network is adopted, in which the BSs are deployed on a hexagonal lattice with density $\lambda_{b}$, denoted as $\Phi_{b}$, and consequently 
TABLE I

SUMMARY OF NOTATIONS

\begin{tabular}{|c|l|}
\hline Symbol & Meaning \\
\hline$\Phi_{e}, \lambda_{e}$ & Process of energy centers and its density. \\
$\Phi_{h}, \lambda_{h}$ & Set of harvesters on a hexagonal lattice and its density. \\
$\Phi_{b}, \lambda_{b}$ & Set of BSs on a hexagonal lattice and its density. \\
$\Phi_{a}, \lambda_{a}$ & $\begin{array}{l}\text { Set of aggregators on a hexagonal lattice and its } \\
\text { density. }\end{array}$ \\
$f(d)$ & Energy decay function of distance $d$. \\
$g(X)$ & Energy intensity at location $X$. \\
$\gamma$ & Maximum energy intensity of the energy field. \\
$\psi$ & Characteristic parameter of the energy field. \\
$\eta$ & Harvester aperture. \\
$B_{0}, P_{0}$ & Typical BS and its transmission power. \\
$C_{0}, K_{0}$ & Cell served by $B_{0}$ and the number of mobiles in the \\
$R_{0, n}, H_{0, n}$ & cell. \\
$p_{\text {out }}, \epsilon$ & Propagation distance and channel coefficient for the \\
$\Delta P_{0}$ & Outage probability and its constraint. \\
$V$ & Reduction of $P_{0}$ due to aggregation loss. \\
$\tau$ & Harvester voltage for power transmission. \\
& Fixed multiplier of $P_{0}$ in $(0,1)$ representing regulated \\
& aggregation loss. \\
\hline
\end{tabular}

the plane is partitioned into hexagonal cells with areas of $1 / \lambda_{b}$. Let $B_{0}, P_{0}$ and $K_{0}$ denote the typical BS, its transmission power and the number of simultaneous mobiles the BS serves, respectively. Since adaptive transmission is effective for counteracting energy spatial randomness as mentioned earlier, $P_{0}$ is assumed to be fixed and equal to the power supplied to the BS so as to keep the exposition concise. In addition, circuit power consumption of each BS is assumed to be negligible compared with $P_{0}{ }^{1}$ Mobiles are assumed to be distributed as a PPP with density $\lambda_{u}$ and all mobiles are scheduled for simultaneous transmissions. Then $K_{0}$ is a Poisson random variable with mean $\lambda_{u} / \lambda_{b}$. A signal transmitted by a BS at $X \in \mathbb{R}^{2}$ with power $P$ is received at a mobile at $Y \in \mathbb{R}^{2}$ with power given as $P H_{X Y}|X-Y|^{-\alpha}$, where $|X-Y|$ is the Euclidean distance between $X$ and $Y, \alpha>2$ the path-loss exponent and the random variable $H_{X Y}$, called a channel coefficient, models fading or shadowing. The channel coefficients are assumed to be i.i.d. Assuming unit noise variance, the received power also gives the received SNR. In the noise-limited network, the condition for reliable data decoding at a mobile is specified by the outage constraint that the received SNR exceeds a given threshold $\theta$ except for a small probability $\epsilon$.

Define an outage event as one that the receive SNR of a typical active mobile is below $\theta$. The outage probability is the network performance metric and defined for the two transmission strategies as follows. First, consider channelindependent transmission, where a BS equally allocates supplied power for transmission to mobiles. Let $R_{0}$ and $H_{0}$

${ }^{1}$ The analysis can be modified to account for fixed BS circuit power, denoted as $\varphi$, by replacing $P_{0}$ with $\left(P_{0}-\varphi\right)$ in the definitions of outage probability in (1) and (2) in the sequel. However, the notation and derivation steps are made tedious while the analytical methods and key insights remain unchanged.

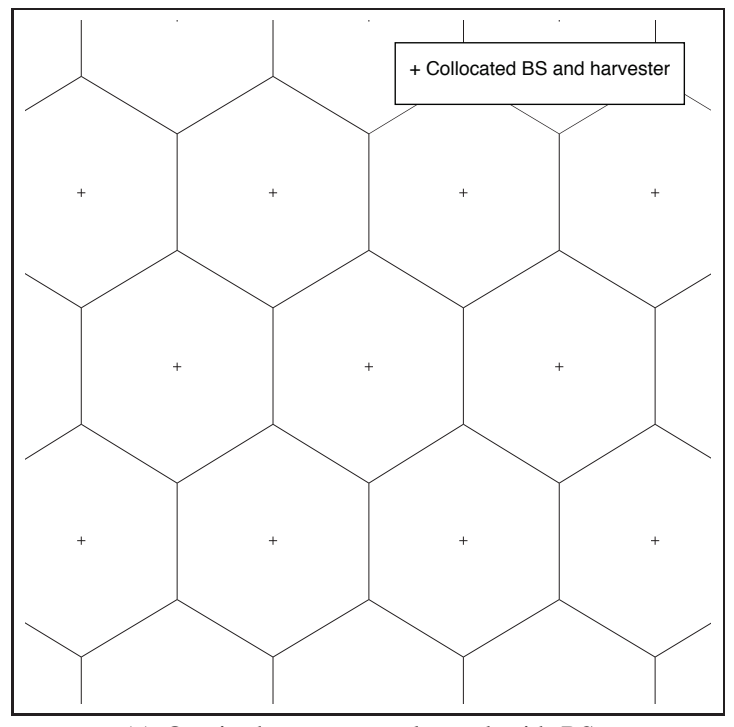

(a) On-site harvesters co-located with BSs.

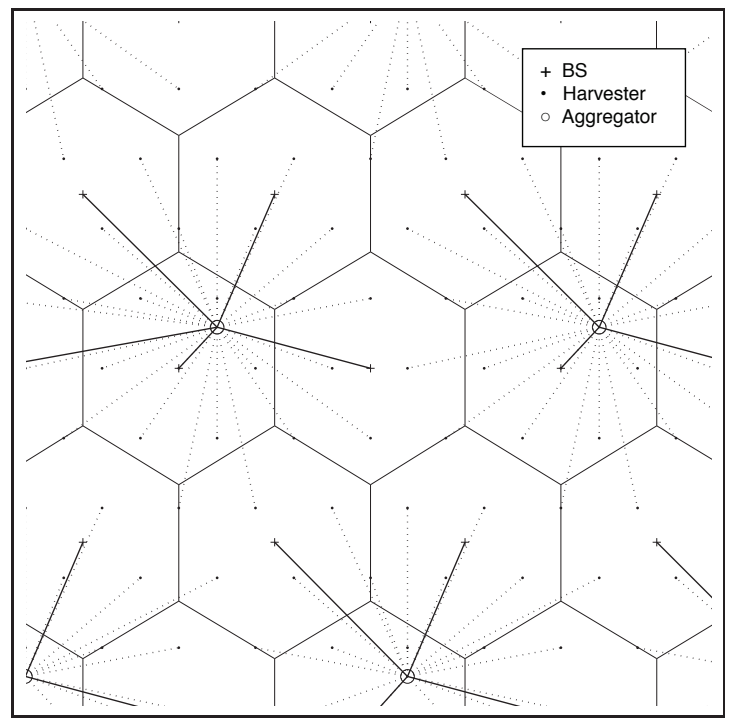

(b) Distributed harvesters.

Fig. 2. Geometric patterns of hexagonal cells, BSs, harvesters and aggregators plotted/marked using solid lines, crosses, dots and circles, respectively. Power transmission lines from harvesters to aggregators and those from aggregators to BSs are plotted using dashed and solid lines, respectively.

denote the propagation distance and the channel coefficient of a typical user in the typical cell, respectively. Then, the outage probability, denoted as $p_{\text {out }}^{\text {id }}$, can be written as

$$
p_{\text {out }}^{\text {id }}=\operatorname{Pr}\left(\frac{P_{0} H_{0} R_{0}^{-\alpha}}{K_{0}}<\theta\right)
$$

where given equal power allocation, the transmission power for each of the $K_{0}$ users in the typical cell is $P_{0} / K_{0}$. Alternatively, the outage probability can be defined with respect to a set of users in the same cell as the probability that the received SNR for least one user is below the threshold. Following similar steps as in the subsequent analysis, one expect the resultant outage probabilities to have similar expressions as those in Propositions 1-4 that arise mainly from the distribution of the energy field instead of the the specific definition of outage 
probability.

Next, consider channel inversion transmission. Transmission power required for each mobile is computed by channel inversion in an attempt to ensure that the receive SNR is equal to $\theta$. Specifically, the $n$-th mobile in the typical cell is under coverage if the allocated transmission power is no smaller than $\theta R_{0, n}^{\alpha} / H_{0, n}$, where $R_{0, n}$ and $H_{0, n}$ represent the corresponding propagation distance and channel coefficient, respectively. It is difficult to write down the outage probability for a typical active mobile, denoted as $p_{\text {out }}^{\text {iv }}$, since it depends on the channels of other active mobiles sharing the BS. However, it can be bounded using the union bound as

$$
p_{\text {out }}^{\text {iv }} \leq \operatorname{Pr}\left(P_{0}<\theta \sum_{n=1}^{K_{0}} \frac{R_{0, n}^{\alpha}}{H_{0, n}}\right) .
$$

The upper bound in (2) is the probability of the event that at least one user is in outage, which is the union of the outage events of individual users.

\section{Energy Field Model ANd Its Properties}

\section{A. Energy Field Model}

The energy field $\Psi$ refers to the set of energy intensities at different locations in the horizontal plane with the maximum denoted as $\gamma>0$. The PPP modeling for the energy centers and its density are denoted as $\Phi_{e} \subset \mathbb{R}^{2}$ and $\lambda_{e}$, respectively. The energy intensity function $g(X)$ is defined for a given location $X \in \mathbb{R}^{2}$ as follows:

$$
g(X)=\gamma \max _{Y \in \Phi_{e}} f(|X-Y|)
$$

where the energy decay function $f$ is

$$
f(d)=e^{-d^{2} / \nu}, \quad d>0 .
$$

Note that $g(X)$ is a Boolean random function [27]. The positive parameter $\nu$ in (4) controls the shape of $f$, thus called the shape parameter, and thereby determines the area of influence of an energy center. Then the characteristic parameter $\psi$ is defined as $\psi=\nu \lambda_{e}$. A large value of $\psi$ corresponds to an energy field with gradual spatial variation and a small value indicates that the field has a lot of "shadows", where energy intensities are much lower than the peak. As observed from the plots in Fig. 3, increasing $\lambda_{e}$ or decreasing $\nu$ introduces more "ripples" in the energy field; the field is almost flat for a large characteristic parameter e.g., $\psi=10$ (or $\lambda_{e}=10$ and $\nu=1)$.

An alternative model of the energy field can result from replacing the max operator in the energy intensity function in (3) with a summation. The two models are shown in Section VI-A to have similar stochastic properties in the operational regime of interest. Furthermore, the energy field based on a different energy decay function is considered in Section VI-B and its effect on the network coverage is analyzed.

\section{B. Energy Field Properties}

First, the distribution function of the energy intensity can be easily obtained by relating it to a Boolean model. To this end, define $r(x)$ as the distance from an energy center to a location with the decayed energy intensity $x$. Then $r(x)$ can be obtained from the equation $f(r(x))=x$ with $f$ in (4) as follows:

$$
r(x)=f^{-1}\left(\frac{x}{\gamma}\right)=\sqrt{\nu \ln \frac{\gamma}{x}} .
$$

Moreover, let $B(X, r)$ denote a disk in $\mathbb{R}^{2}$ centered at $X$ and with a radius $r$. The region of the energy field where energy intensities exceed a threshold $x$ corresponds to a Boolean model $\bigcup_{X \in \Phi_{e}} B(X, r(x))$. Then for given $X$ and $x \in[0, \gamma]$, the distribution function of $g(X)$ can be written in terms of the model and obtained as

$$
\begin{aligned}
\operatorname{Pr}(g(X) \leq x) & =\operatorname{Pr}\left(X \notin \bigcup_{X \in \Phi_{e}} B(X, r(x))\right) \\
& =e^{-\pi \lambda_{e} r^{2}(x)} \\
& =\left(\frac{x}{\gamma}\right)^{\pi \psi}
\end{aligned}
$$

where the last equality is obtained by substituting (5) and using the definition of $\psi$. Next, the mean and variance of the energy intensity function can be directly derived using the distribution function in (6) as follows:

$$
\begin{aligned}
\mathrm{E}[g(X)] & =\frac{\pi \psi \gamma}{1+\pi \psi}, \\
\mathrm{E}\left[g^{2}(X)\right] & =\frac{\pi \psi \gamma^{2}}{2+\pi \psi}, \\
\operatorname{var}(g(X)) & =\frac{\pi \psi \gamma^{2}}{(2+\pi \psi)(1+\pi \psi)^{2}} .
\end{aligned}
$$

As $\psi \rightarrow \infty, \mathrm{E}[g(X)]$ is seen to converge to $\gamma$ while $\operatorname{var}(g(X))$ diminishes inversely with $\psi^{2}$. On the other hand, as $\psi \rightarrow 0$, both quantities become proportional to $\psi$.

Last, the joint distribution of the energy intensities $g\left(X_{1}\right)$ and $g\left(X_{2}\right)$ at two different locations $X_{1}$ and $X_{2}$ is derived. They are independent if $\left|X_{1}-X_{2}\right| \geq r\left(x_{1}\right)+r\left(x_{2}\right)$ :

$$
\begin{aligned}
\operatorname{Pr}\left(g\left(X_{1}\right) \leq x_{1}, g\left(X_{2}\right) \leq x_{2}\right)= & \operatorname{Pr}\left(g\left(X_{1}\right) \leq x_{1}\right) \times \\
& \operatorname{Pr}\left(g\left(X_{2}\right) \leq x_{2}\right) \\
= & \left(\frac{x_{1} x_{2}}{\gamma^{2}}\right)^{\pi \psi} .
\end{aligned}
$$

$$
\text { If } \begin{aligned}
\left|X_{1}-X_{2}\right| & <r\left(x_{1}\right)+r\left(x_{2}\right), \\
\operatorname{Pr}\left(g\left(X_{1}\right)\right. & \left.\leq x_{1}, g\left(X_{2}\right) \leq x_{2}\right) \\
= & \left(\frac{x_{1} x_{2}}{\gamma^{2}}\right)^{\pi \nu \lambda_{e}} e^{\lambda_{e}\left|B\left(X_{1}, r\left(x_{1}\right)\right) \cap B\left(X_{2}, r\left(x_{2}\right)\right)\right|}
\end{aligned}
$$

where the operator $|\cdot|$ applied on a set gives its measure (area). For ease of notation, rewrite $r\left(x_{1}\right)$ and $r\left(x_{2}\right)$ as $r_{1}$ and $r_{2}$ and define $d=\left|X_{1}-X_{2}\right|$. The area of the overlapping region 


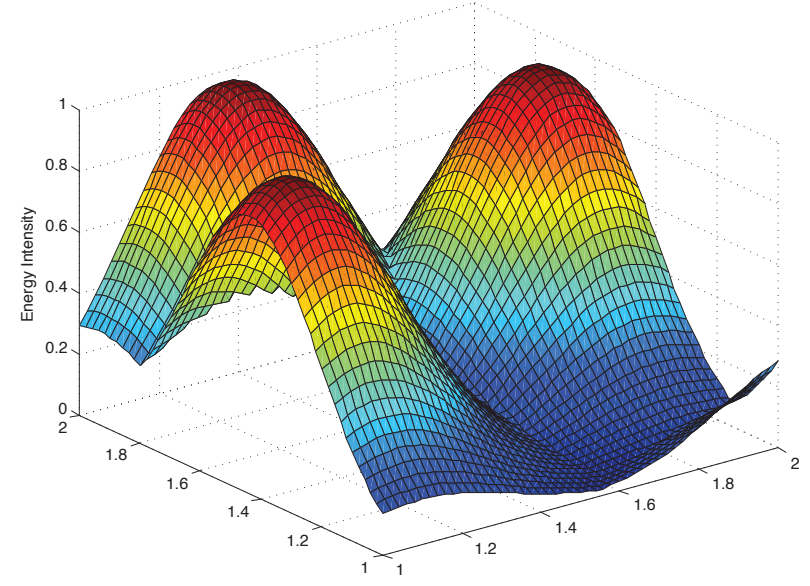

(a) $\lambda_{e}=1, \nu=0.1$

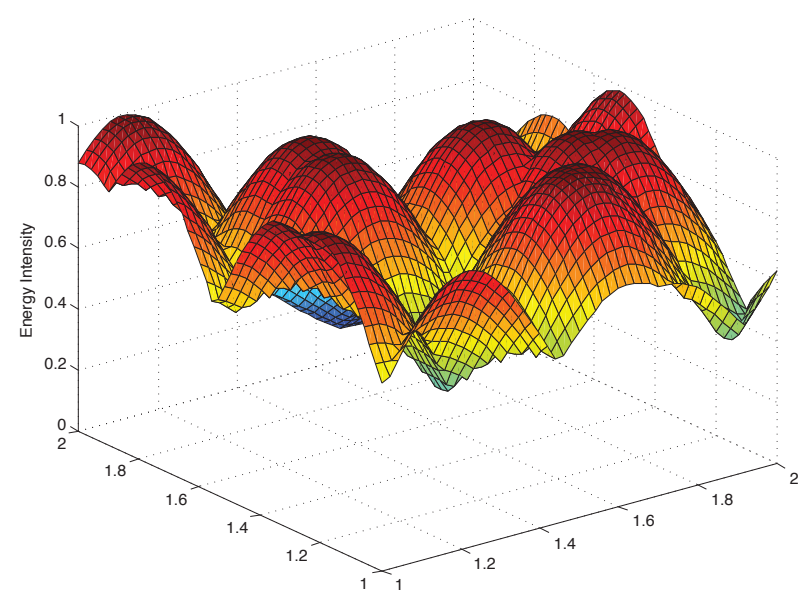

(c) $\lambda_{e}=10, \nu=0.1$

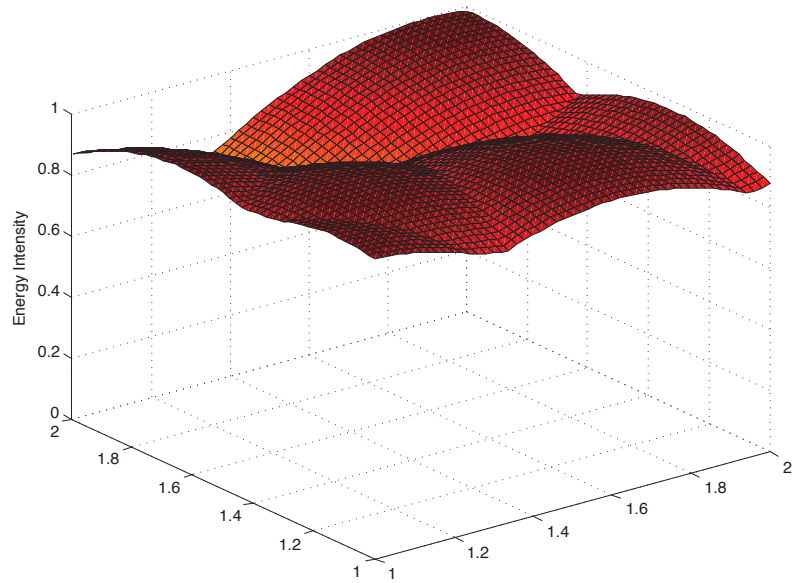

(b) $\lambda_{e}=1, \nu=1$

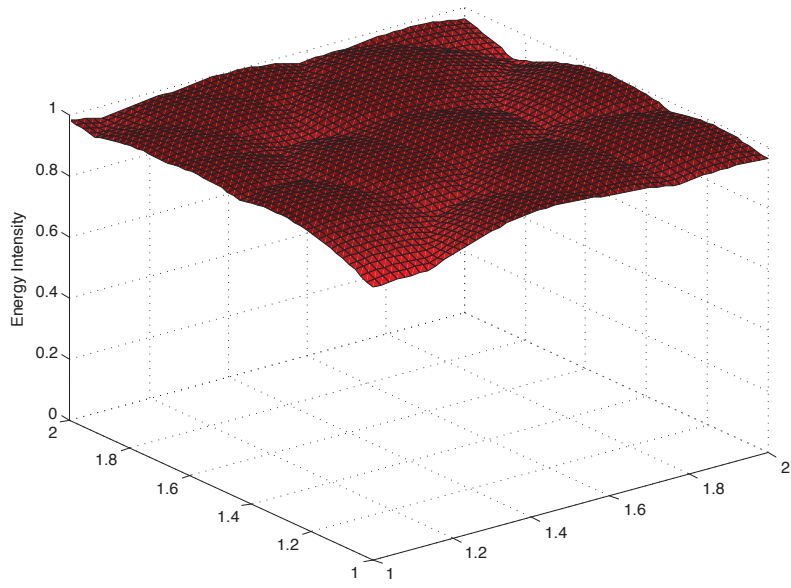

(d) $\lambda_{e}=10, \nu=1$

Fig. 3. Energy field for different combinations of the energy center density $\lambda_{e}$ and shape parameter $\nu$ and different realizations of the energy-center process.

between $B\left(X_{1}, r_{1}\right)$ and $B\left(X_{2}, r_{2}\right)$ is known to be given as

$$
\begin{aligned}
& \left|B\left(X_{1}, r_{1}\right) \cap B\left(X_{2}, r_{2}\right)\right|=r_{1}^{2} \cos ^{-1}\left(\frac{r_{1}^{2}+d^{2}-r_{2}^{2}}{2 d r_{1}}\right)+ \\
& +r_{2}^{2} \cos ^{-1}\left(\frac{r_{2}^{2}+d^{2}-r_{1}^{2}}{2 d r_{2}}\right)-d \sqrt{r_{1}^{2}-\left(\frac{r_{1}^{2}+d^{2}-r_{2}^{2}}{2 d}\right)^{2}} .
\end{aligned}
$$

For the special case of $x_{1}=x_{2}=x$ (thus $r_{1}=r_{2}=r$ ),

$$
\operatorname{Pr}\left(g\left(X_{1}\right) \leq x, g\left(X_{2}\right) \leq x\right)=\left(\frac{x}{\gamma}\right)^{2 \pi \nu \lambda_{e}(1-\Delta(d / r))}
$$

where $\Delta(d / r) \geq 0$ and

$$
\Delta(y)=\frac{1}{2 \pi} \cos ^{-1}\left(\frac{y}{2}\right)-\frac{y}{4 \pi} \sqrt{4-y^{2}} .
$$

Comparing (9) and (10), one can see that reducing the distance between two locations increases the joint cumulative distribution function of the corresponding energy intensities as they become more correlated.

\section{Network Coverage with ON-Site Harvesters}

\section{A. Network Coverage with Channel-Independent Transmis-} sion

To facilitate analysis, the outage probability is decomposed as $p_{\text {out }}^{\text {id }}=p_{b}+p_{a}$ with $p_{b}$ and $p_{a}$ defined as

$$
\begin{aligned}
p_{a}= & \operatorname{Pr}\left(\frac{P_{0} H_{0} R_{0}^{-\alpha}}{K_{0}}<\theta \mid K_{0} H_{0}^{-1} R_{0}^{\alpha} \leq \frac{\eta \gamma}{\theta}\right) \times \\
& \operatorname{Pr}\left(K_{0} H_{0}^{-1} R_{0}^{\alpha} \leq \frac{\eta \gamma}{\theta}\right), \\
p_{b}= & \operatorname{Pr}\left(K_{0} H_{0}^{-1} R_{0}^{\alpha}>\frac{\eta \gamma}{\theta}\right) .
\end{aligned}
$$

The component $p_{a}$ is the probability that the energy intensity at the typical BS site is so low as to cause an outage event at the typical mobile even though the maximum intensity is sufficiently high for avoiding such an event. The other component $p_{b}$ represents the probability that the maximum energy intensity at the typical BS is insufficiently high for avoiding an outage event at the typical mobile, which occurs due to the combined effect of the number of simultaneous users $K_{0}$ being too large, the channel gain being too small, and the propagation distance $R_{0}$ being too long. In other 
words, $p_{a}$ and $p_{b}$ are the components of outage probability arising from the energy field spatial variation and the limit on the maximum harvested power, respectively. The outage probability is characterized by analyzing $p_{a}$ and $p_{b}$ separately.

To this end, it is useful to define a random variable $\bar{R}_{0}$ as $\bar{R}_{0}=\sqrt{\lambda_{b}} R_{0}$ that gives the propagation distance of a mobile uniformly distributed in a cell of unit area. Note that a hexagon of unit area can be outer bounded by a disk with the area of $\frac{2 \pi}{3 \sqrt{3}}$. Let $D$ denote the distance from the center of the disk to a random point uniformly distributed in the disk. Then

$$
\begin{aligned}
\operatorname{Pr}\left(\bar{R}_{0} \leq x\right) & \geq \operatorname{Pr}(D \leq x) \\
& =\frac{3 \sqrt{3}}{2} x^{2}, \quad 0 \leq x \leq \sqrt{\frac{2}{3 \sqrt{3}}} .
\end{aligned}
$$

The probability $p_{a}$ is shown in the following lemma to be an exponential function of the energy field characteristic parameter $\psi$, when $\psi$ is sufficiently small. The proof of the lemma is provided in Appendix A.

Lemma 1. The probability $p_{a}$ can be upper bounded as

$$
p_{a} \leq \frac{2}{2+\alpha \pi \psi}\left(\frac{c_{2} \theta \mathrm{E}\left[H_{0}^{-1}\right] \lambda_{u}}{\gamma \eta \lambda_{b}^{1+\frac{\alpha}{2}}}\right)^{\min (\pi \psi, 1)}
$$

where $c_{2}=\left(\frac{2}{3 \sqrt{3}}\right)^{\frac{\alpha}{2}}$.

One can observe from (12) that $p_{b}$ is the tail probability of the product of multiple random variables and thus it is difficult to derive a closed-form expression for the probability. Following typical approaches, we apply a probabilistic inequality, namely Markov's inequality, to obtain an upper bound on $p_{b}$ and large deviation theory to characterize its asymptotic scaling. The following lemma is obtained based on Markov's inequality with the proof in Appendix B.

Lemma 2. The probability $p_{b}$ in (12) can be upper bounded as

$$
p_{b} \leq \frac{c_{3} \theta \lambda_{u} \mathrm{E}\left[H_{0}^{-1}\right]}{\eta \gamma \lambda_{b}^{1+\frac{\alpha}{2}}}
$$

where the constant $c_{3}=\frac{2}{2+\alpha}\left(\frac{2}{3 \sqrt{3}}\right)^{\frac{\alpha}{2}}$.

Given $p_{\text {out }}^{\text {id }}=p_{b}+p_{a}$, combining the results in Lemmas 1 and 2 leads to the following first main result as follows.

Proposition 1. Consider the scenario where BSs adopt channel-independent transmission and are powered by on-site harvesters. The outage probability can be bounded as

$p_{\text {out }}^{\text {id }} \leq \frac{2}{2+\alpha \pi \psi}\left(\frac{c_{2} \theta \mathrm{E}\left[H_{0}^{-1}\right] \lambda_{u}}{\lambda_{b}^{1+\frac{\alpha}{2}} \gamma \eta}\right)^{\min (\pi \psi, 1)}+\frac{c_{3} \theta \lambda_{u} \mathrm{E}\left[H_{0}^{-1}\right]}{\lambda_{b}^{1+\frac{\alpha}{2}} \eta \gamma}$.

Consider the scenario where the maximum harvested power $\gamma \eta$ is large and the characteristic parameter $\psi$ is small. Then the outage probability decreases monotonically with these parameters following the scaling law of

$$
p_{\text {out }}^{\text {id }} \propto(\gamma \eta)^{-\pi \psi}
$$

which is verified by simulation in the sequel.
Next, $p_{b}$ is analyzed using large deviation theory. To this end, it is necessary to consider a specific type of distribution for the channel coefficient $H_{0}$. Let $\bar{F}$ denote the complementary cumulative distribution function (CCDF) of the random variable (RV) $H_{0}^{-1}$ in (12). Assume that $H_{0}^{-1}$ is a regularly varying random variable defined by the following condition:

$$
\lim _{t \rightarrow \infty} \frac{\bar{F}(\zeta t)}{\bar{F}(t)}=\zeta^{-\omega}
$$

where $\omega>0$ is the exponent of the distribution and $\zeta>1$. One example is that $H_{0}$ has the chi-squared distribution that is typical for wireless channels:

$$
\operatorname{Pr}\left(H_{0}<t\right)=\frac{1}{\Gamma(\omega)} \int_{0}^{t} x^{\omega-1} e^{-x} d x, \quad t \geq 0
$$

where $\omega>0$ is a positive integer and the distribution parameter and $\Gamma$ denotes the gamma function. Then $H_{0}^{-1}$ is a regularly varying RV with the exponent $\omega$. Based on the assumption and applying Breiman's Theorem (see Lemma 7 in Appendix C), an asymptotic bound on $p_{b}$ is obtained as shown below.

Lemma 3. Suppose that the inverse of a channel coefficient is a regularly varying $R V$. As $\eta \gamma \rightarrow \infty$, the probability $p_{b}$ is bounded $a s^{2}$

$$
p_{b} \preceq \mathrm{E}\left[K_{0}^{\omega}\right] \operatorname{Pr}\left(H_{0} \leq \frac{\left(\frac{2 \lambda_{b}}{3 \sqrt{3}}\right)^{\frac{\alpha}{2}} \theta}{\eta \gamma}\right), \quad \eta \gamma \rightarrow \infty
$$

where $\mathrm{E}\left[K_{0}^{\omega}\right]$ is given as

$$
\mathrm{E}\left[K_{0}^{\omega}\right]=\sum_{m=1}^{\omega}\left(\frac{\lambda_{u}}{\lambda_{b}}\right)^{m} \frac{1}{m} \sum_{k=0}^{m}(-1)^{m-k}\left(\begin{array}{c}
m \\
k
\end{array}\right) k^{\omega} .
$$

In particular, if a channel coefficient is a chi-squared $R V$ with parameter $\omega$,

$p_{b} \preceq \mathrm{E}\left[K_{0}^{\omega}\right]\left(\frac{\eta \gamma}{\left(\frac{2 \lambda_{b}}{3 \sqrt{3}}\right)^{\frac{\alpha}{2}} \theta}\right)^{-\omega}+O\left((\eta \gamma)^{-\omega-1}\right), \quad \eta \gamma \rightarrow \infty$.

Remark: It is possible to derive asymptotic upper bounds on $p_{b}$ for other types of channel coefficient distribution. For instance, [28, Theorem 2.1] can be applied to obtain a bound for the sub-exponential distribution defined by the condition $\lim _{x \rightarrow \infty} \frac{\overline{F * F}(x)}{F(x)}=2$ where ' $*$ ' denotes convolution. Such results will change the second but not the first term of the upper bound on $p_{\text {out }}^{\text {id }}$ in Proposition 2 presented shortly.

Combining the results in Lemmas 1 and 3 leads to the second main result as follows:

Proposition 2. Consider the scenario where BSs adopt channel-independent transmission and are powered by on-site harvesters. Suppose that the inverse of a channel coefficient is

\footnotetext{
${ }^{2}$ Two asymptotic relation operators, " $"$ and " $\preceq "$, are defined as follows. Two functions $h(x)$ and $q(x)$ are asymptotically equivalent, denoted as $h(x) \sim g(x)$, if $\lim _{x \rightarrow \infty} \frac{h(x)}{g(x)}=1$. The case of $\lim _{x \rightarrow \infty} \frac{h(x)}{q(x)} \leq 1$ is represented by $h(x) \preceq q(x)$.
} 
a regularly varying $R V$, the outage probability can be bounded as $\eta \gamma \rightarrow \infty$ as follows:

$$
\begin{aligned}
p_{\text {out }}^{\text {id }} \preceq & \frac{2}{2+\alpha \pi \psi}\left(\frac{\lambda_{b}^{1+\frac{\alpha}{2}} \gamma \eta}{c_{2} \theta \mathrm{E}\left[H_{0}^{-1}\right] \lambda_{u}}\right)^{-\min (\pi \psi, 1)}+ \\
& \mathrm{E}\left[K_{0}^{\omega}\right]\left(\left(\frac{2 \lambda_{b}}{3 \sqrt{3}}\right)^{\frac{\alpha}{2}} \theta\right)^{\omega} \operatorname{Pr}\left(H_{0}<\frac{1}{\eta \gamma}\right) .
\end{aligned}
$$

In particular, if channel coefficients follow i.i.d. chi-squared distributions, as $\eta \gamma \rightarrow \infty$,

$$
\begin{aligned}
p_{\text {out }}^{\text {id }} \preceq & \frac{2}{2+\alpha \pi \psi}\left(\frac{\lambda_{b}^{1+\frac{\alpha}{2}} \gamma \eta}{c_{2} \theta \Gamma(\omega-1) \lambda_{u}}\right)^{-\min (\pi \psi, 1)}+ \\
& \frac{\mathrm{E}\left[K_{0}^{\omega}\right]}{\Gamma(\omega+1)}\left(\frac{\eta \gamma}{\left(\frac{2 \lambda_{b}}{3 \sqrt{3}}\right)^{\frac{\alpha}{2}} \theta}\right)^{-\omega}+O\left((\eta \gamma)^{-\omega-1}\right) .
\end{aligned}
$$

Comparing the results in Propositions 1 and 2, both upper bounds on $p_{\text {out }}^{\text {id }}$ have identical first term, which is dominant when $\eta \gamma$ is large. The second term in (20), proportional to $(\gamma \eta)^{-\omega}$, decays faster than the counterpart in Proposition 1, proportional to $(\gamma \eta)^{-1}$, when $\omega>1$. This is due to a tighter bound on $p_{b}$ derived using large deviation theory with respect to that obtained using Markov's inequality.

\section{B. Network Coverage with Channel Inversion Transmission}

Following the same method as in the preceding subsection, the outage probability in (2) can be decomposed as $p_{\text {out }}^{\text {iv }} \leq$ $p_{c}+p_{d}$ where

$$
\begin{gathered}
p_{c}=\operatorname{Pr}\left(P_{0}<\theta \sum_{n=1}^{K_{0}} \frac{R_{0, n}^{\alpha}}{H_{0, n}} \mid \sum_{n=1}^{K_{0}} \frac{R_{0, n}^{\alpha}}{H_{0, n}} \leq \frac{\eta \gamma}{\theta}\right) \times \\
\operatorname{Pr}\left(\sum_{n=1}^{K_{0}} \frac{R_{0, n}^{\alpha}}{H_{0, n}} \leq \frac{\eta \gamma}{\theta}\right), \\
p_{d}=\operatorname{Pr}\left(\sum_{n=1}^{K_{0}} \frac{R_{0, n}^{\alpha}}{H_{0, n}}>\frac{\eta \gamma}{\theta}\right) .
\end{gathered}
$$

Their key difference from those of their counterparts $p_{b}$ and $p_{a}$ in the preceding section is that the transmission power of a typical BS for the current case is a compound Poisson random variable. However, the expected transmission powers for both cases are identical due to the following equality:

$$
\mathrm{E}\left[\sum_{n=1}^{K_{0}} \frac{R_{0, n}^{\alpha}}{H_{0, n}}\right]=\mathrm{E}\left[K_{0}\right] \mathrm{E}\left[R_{0, n}^{\alpha}\right] \mathrm{E}\left[H_{0, n}^{-1}\right] .
$$

Given this equality, the bounds on $p_{a}$ in (11) and $p_{b}$ in (12) can be shown to hold for $p_{c}$ in (20) and $p_{d}$ in (21), respectively, yielding the following proposition.

Proposition 3. Consider the scenario of on-site harvesters. The upper bound on the outage probability for the case of channel-independent transmission as given in Proposition 1 also holds for the case of channel inversion transmission.

Despite the identical upper bounds, the outage probability for channel inversion transmission is lower than that for channel-independent transmission. The performance gain is due to adapting transmission-power allocation to multiuser channel states to minimize the number of mobiles in outage.

Next, $p_{c}$ is analyzed using large deviation theory. To this end, some useful definitions and results are introduced as follows. The distribution of a $\mathrm{RV} X$ has a light tail if $\mathrm{E}\left[e^{\delta X}\right]<\infty$ for some $\delta>0$ and a heavy tail if $\mathrm{E}\left[e^{\delta X}\right]=\infty$ for all $\delta>0$. The following result is from [29, Theorem 2].

Lemma 4. Let $\left\{X_{m}\right\}$ be a set of i.i.d. random variables having a heavy-tailed distribution and $N$ have a light-tailed distribution independent of those of $\left\{X_{m}\right\}$. Then

$$
\operatorname{Pr}\left(\sum_{m=1}^{N} X_{m}>t\right) \sim \mathrm{E}[N] \operatorname{Pr}\left(X_{1}>t\right) \quad \text { as } t \rightarrow \infty .
$$

To apply the result, the upper bound on $p_{d}$ in (21) is simplified using the inequality $R_{0, n} \leq \sqrt{2 \lambda_{b} / 3 \sqrt{3}}$ as

$$
p_{d} \leq \operatorname{Pr}\left(\sum_{n=1}^{K_{0}} H_{0, n}^{-1}>\frac{\eta \gamma}{\left(2 \lambda_{b} / 3 \sqrt{3}\right)^{\frac{\alpha}{2}} \theta}\right) \text {. }
$$

One can see that the upper bound is the tail probability of a compound Poisson RV, $\sum_{n=1}^{K_{0}} H_{0, n}^{-1}$. Note that the Poisson distribution of $K_{0}$ has a light tail since it decays faster than the exponential rate as observed from the following inequality (see e.g., [30, Theorem 5.4]):

$$
\operatorname{Pr}\left(K_{0} \geq x\right) \leq \frac{e^{-\lambda_{m} / \lambda_{b}}\left(e \lambda_{m} / \lambda_{b}\right)^{x}}{x^{x}}, \quad \text { if } x>\lambda_{m} / \lambda_{b} .
$$

Then the lemma below follows from (23) and Lemma 4, which is the channel inversion counterpart of Lemma 3.

Lemma 5. Suppose that the inverse of a channel coefficient has a heavy-tailed distribution. As $\eta \gamma \rightarrow \infty$, the probability $p_{d}$ is bounded as

$$
p_{d} \preceq \frac{\lambda_{u}}{\lambda_{b}} \operatorname{Pr}\left(H_{0} \leq \frac{\left(\frac{2 \lambda_{b}}{3 \sqrt{3}}\right)^{\frac{\alpha}{2}} \theta}{\eta \gamma}\right), \quad \eta \gamma \rightarrow \infty
$$

In particular, if a channel coefficient is a chi-squared RV with parameter $\omega$,

$$
p_{d} \preceq \frac{\lambda_{u}}{\lambda_{b}}\left(\frac{\eta \gamma}{\left(\frac{2 \lambda_{b}}{3 \sqrt{3}}\right)^{\frac{\alpha}{2}} \theta}\right)^{-\omega}+O\left((\eta \gamma)^{-\omega-1}\right), \quad \eta \gamma \rightarrow \infty .
$$

As mentioned, the bound on $p_{a}$ in (11) holds for $p_{c}$ in (20). Combining this result, the bounds on $p_{d}$ in Lemma 5 and the inequality $p_{\text {out }}^{\text {iv }} \leq p_{c}+p_{d}$ gives the following result.

Proposition 4. Consider the scenario where BSs adopt channel inversion transmission and are powered by on-site harvesters. Suppose that the inverse of a channel coefficient is a heavy-tailed $R V$, the outage probability can be bounded as follows: as $\eta \gamma \rightarrow \infty$,

$$
\begin{gathered}
p_{\text {out }}^{i v} \preceq \frac{2}{2+\alpha \pi \psi}\left(\frac{\lambda_{b}^{1+\frac{\alpha}{2}} \gamma \eta}{c_{2} \theta \mathrm{E}\left[H_{0}^{-1}\right] \lambda_{u}}\right)^{-\min (\pi \psi, 1)}+ \\
\frac{\lambda_{u}}{\lambda_{b}} \operatorname{Pr}\left(H_{0}<\left(\frac{2 \lambda_{b}}{3 \sqrt{3}}\right)^{\frac{\alpha}{2}} \frac{\theta}{\eta \gamma}\right) .
\end{gathered}
$$


In particular, if channel coefficients follow i.i.d. chi-squared distributions, as $\eta \gamma \rightarrow \infty$,

$$
\begin{aligned}
p_{\text {out }}^{i v} \preceq & \frac{2}{2+\alpha \pi \psi}\left(\frac{\lambda_{b}^{1+\frac{\alpha}{2}} \gamma \eta}{c_{2} \theta \Gamma(\omega-1) \lambda_{u}}\right)^{-\min (\pi \psi, 1)}+ \\
& \frac{\lambda_{u}}{\Gamma(\omega+1) \lambda_{b}}\left(\frac{\eta \gamma}{\left(\frac{2 \lambda_{b}}{3 \sqrt{3}}\right)^{\frac{\alpha}{2}} \theta}\right)^{-\omega}+O\left((\eta \gamma)^{-\omega-1}\right) .
\end{aligned}
$$

Comparing the results in Propositions 2 and 4, the outage probability for channel inversion transmission follows the same scaling laws as those for channel-independent transmission except for some difference in linear factors. As a result, $p_{\text {out }}^{\text {iv }}$ is smaller than $p_{\text {out }}^{\text {id }}$, as also observed in the simulation results in the sequel.

\section{Network Coverage with Distributed HARVESTERS}

\section{A. Network Coverage}

In this section, it is shown that aggregating energy harvested by many distributed harvesters stabilizes the power supplied to the BSs by the law of large numbers. It is assumed that the energy aggregation loss is regulated such that the scaling factor of BS transmission powers due to such loss is smaller than a constant $\tau \in(0,1)$. The design requirement under this constraint is analyzed in the next subsection. The harvester lattice partitions the plane into small hexagonal regions, each with area of $1 / \lambda_{h}$. Whether each region contains an energy center can be indicated by a set of independent Bernoulli random variables $\left\{Q_{n}\right\}$ with probabilities $\exp \left(-\lambda_{e} / \lambda_{h}\right)$ and $\left[1-\exp \left(-\lambda_{e} / \lambda_{h}\right)\right]$ for the values of 0 and 1 , respectively. Despite the independence of the numbers of energy center in different regions, it is important to note that the energy intensities at harvesters are correlated [see their joint distribution in (8) and (9)]. The energy intensity at each harvester is at least $\exp \left(-\frac{2}{3 \sqrt{3} \nu \lambda_{h}}\right)$ if the corresponding small region contains an energy center or otherwise takes on some positive value. Based on the above points, the transmission power of the typical BS is lower bounded as

$$
\begin{aligned}
P_{0} & \geq \frac{\tau \gamma \eta \lambda_{a}}{\lambda_{b}} \sum_{n=1}^{M_{0}} Q_{n} e^{\left(-\frac{2}{3 \sqrt{3} \nu \lambda_{h}}\right)} \\
& =\frac{\tau \gamma \eta \lambda_{h}}{\lambda_{b}} \times \frac{\lambda_{a} M_{0}}{\lambda_{h}} \times \frac{1}{M_{0}} \sum_{n=1}^{M_{0}} Q_{n} e^{-\frac{2}{3 \sqrt{3} \nu \lambda_{h}}}
\end{aligned}
$$

where $M_{0}$ represents the number of harvesters connected to the typical aggregator. Consider the scenario of sparse aggregators with each connected to many harvesters, corresponding to $\lambda_{a} \rightarrow 0$. As a result, $M_{0} \rightarrow \infty$ and $\lambda_{a} M_{0} / \lambda_{h} \rightarrow 1$. Then it follows from (25) that

$\lim _{\lambda_{a} \rightarrow 0} P_{0}\left(\lambda_{a}\right) \geq \frac{\tau \gamma \eta \lambda_{h}}{\lambda_{b}} \lim _{M_{0} \rightarrow \infty} \frac{1}{M_{0}} \sum_{n=1}^{M_{0}} Q_{n} e^{\left(-\frac{2}{3 \sqrt{3} \nu \lambda_{h}}\right)}$.

The law of large numbers gives the following lemma.
Lemma 6. With the harvester density $\lambda_{h}$ fixed, as the aggregator density $\lambda_{a} \rightarrow 0$, the transmission power for the typical $B S$ converges as

$$
\lim _{\lambda_{a} \rightarrow 0} P_{0}\left(\lambda_{a}\right) \geq \frac{\tau \gamma \eta \lambda_{h}}{\lambda_{b}}\left(1-e^{-\frac{\lambda_{e}}{\lambda_{h}}}\right) e^{-\frac{2}{3 \sqrt{3} \nu \lambda_{h}}}, \quad \text { a.s. }
$$

In other words, $P_{0}$ is lower bounded by a constant and thus its randomness due to energy spatial variation diminishes. If the energy centers are dense $\left(\lambda_{e} \gg \lambda_{h}\right)$ and the shape parameter $\nu$ is large $\left(\nu \lambda_{h} \gg 1\right)$, the transmission power approaches its upper bound $\gamma \eta \lambda_{h} / \lambda_{b}$.

The power stabilization by the spatial averaging of the energy field removes one random variable from the outage probability. Consider the case of channel-independent transmission and the corresponding outage probability in (1). Using Lemma 6 and applying Markov's inequality, the outage probability for small aggregator density can be bounded as

$$
\lim _{\lambda_{a} \rightarrow 0} p_{\text {out }}^{\text {id }}\left(\lambda_{a}\right) \leq \frac{\theta \lambda_{b} \mathrm{E}\left[H^{-1}\right] \mathrm{E}[K] \mathrm{E}\left[R^{\alpha}\right]}{\tau \gamma \eta \lambda_{h}\left(1-e^{-\frac{\lambda_{e}}{\lambda_{h}}}\right) e^{-\frac{2}{3 \sqrt{3} \nu \lambda_{h}}}} .
$$

Substituting $\mathrm{E}[K]=\lambda_{u} / \lambda_{b}$ and the result in (48) yields the result in Proposition 5 as given below. The result is proved to also hold for channel-inversion transmission using (2) and following a similar procedure.

Proposition 5. With the harvester density $\lambda_{h}$ fixed, as the aggregator density $\lambda_{a} \rightarrow 0$, the outage probabilities for both the channel-independent and channel inversion transmissions can be bounded as

$$
\lim _{\lambda_{a} \rightarrow 0} p_{\text {out }}^{\text {id }}\left(\lambda_{a}\right), \lim _{\lambda_{a} \rightarrow 0} p_{\text {out }}^{i v}\left(\lambda_{a}\right) \leq \frac{c_{3} \theta \mathrm{E}\left[H^{-1}\right] \lambda_{u}}{\tau \gamma \eta \lambda_{b}^{\frac{\alpha}{2}}} \lambda_{h}\left(1-e^{-\frac{\lambda e}{\lambda_{h}}}\right) e^{-\frac{2}{3 \sqrt{3} \nu \lambda_{h}}} .
$$

The results in Proposition 5 suggest that

$$
\lim _{\lambda_{a} \rightarrow 0} p_{\text {out }}^{\text {id }}\left(\lambda_{a}\right), \lim _{\lambda_{a} \rightarrow 0} p_{\text {out }}^{\text {iv }}\left(\lambda_{a}\right) \propto \frac{1}{\gamma \eta} \times \frac{1}{\lambda_{h} / \lambda_{b}} \times \frac{\lambda_{u}}{\lambda_{b}} \times \lambda_{b}^{-\frac{\alpha}{2}}
$$

where the factors represent in order the inverses of the maximum power generated by a single harvester, the number of harvesters per BS, the expected number of active mobiles per cell, and the expected propagation loss.

\section{B. Energy Aggregation Loss}

It is well known that the power line loss, $\Delta P$, for transmitting the power of $P$ to a receiver is given as

$$
\Delta P=\frac{\beta P^{2} d}{V^{2}}
$$

where $V$ is the voltage and $\beta$ is a constant depending on the power line characteristics, such as resistivity and cross-section area. In other words, the total transmission power is $(P+\Delta P)$. Consider an arbitrary harvester in a typical cluster. Let $V_{0}, g_{0}$, and $d_{0}$ denote the transmission voltage and energy intensity at this harvester and the corresponding distance for power transmission to the connected aggregator, respectively. Then the power harvested at and transmitted by the harvester is $\eta g_{0}$. By the definition of transmission efficiency $\tau$, the transmission 
loss, denoted as $\Delta P_{0}$, should be no larger than the fraction $(1-\tau)$ of transmission power:

$$
\Delta P_{0} \leq(1-\tau) \eta g_{0} .
$$

Since the power effectively transmitted from the harvester to the aggregator is $\tau \eta g_{0}$, it follows from (29) that

$$
\Delta P_{0}=\frac{\beta\left(\tau \eta g_{0}\right)^{2}}{V_{0}^{2}} d_{0} .
$$

Combining (30) and (31) leads to a requirement on the voltage

$$
V_{0} \geq \tau \sqrt{\frac{\beta \eta g_{0} d_{0}}{1-\tau}} .
$$

The distance $d_{0}$ can be bounded as $d_{0} \leq \sqrt{\frac{2}{3 \sqrt{3} \lambda_{a}}}$ since the harvester lies in a hexagon centered at the connected aggregator and with an area of $1 / \lambda_{a}$. Using this bound as well as $g_{0} \leq \gamma$, a sufficient condition for meeting the requirement in (32) is obtained as shown in the following proposition.

Proposition 6. Consider distributed harvesters and aggregators with fixed densities. A sufficient condition on the harvester voltage for achieving a given transfer efficiency $\tau \in(0,1)$ is

$$
V_{0}=\tau \sqrt{\frac{\beta \eta \gamma}{1-\tau} \sqrt{\frac{2}{3 \sqrt{3}}}} \lambda_{a}^{-\frac{1}{4}}
$$

Given fixed $\tau$, one can observe from Proposition 6 that as the size of harvester clusters increases by letting $\lambda_{a} \rightarrow 0$, it is sufficient for the harvester voltage to scale as $\lambda_{a}^{-1 / 4}$ multiplied by a constant, resulting in reliable power supply to all BSs.

It is also interesting to consider the case with fixed $V_{0}$ for which there exists a conflict between suppressing energy spatial randomness by energy aggregation and reducing the resultant power-transmission loss. Specifically, based on (33), decreasing the aggregator density $\lambda_{a}$ reduces the transmission efficiency $\tau$, which in turn increases the outage-probability upper bound in Proposition 5. This makes it necessary to optimize $\lambda_{a}$ for minimizing the outage probability. Solving the problem is non-trivial and outside the scope of this paper but warrants further investigation.

\section{EXTENSIONS AND DISCUSSION}

\section{A. Shot-Noise Based Energy Field Model}

In this subsection, the energy field model proposed in Section III is compared with an alternative model described as follows. By replacing the max operator in the energy intensity function in (3) with a summation, the result, denoted as $\tilde{g}(X)$, represents spatial interpolation of the energy centers:

$$
\tilde{g}(X)=\gamma \sum_{Y \in \Phi_{e}} f(|X-Y|)
$$

One drawback of the resultant alternative model is that the maximum energy intensity of the field can no longer be retained as $\gamma$ as it becomes a random variable with unbounded support, which is impractical. As another drawback, the random function in (34) is known as a shot-noise process and its distribution function has no simple form [31].
The expectation of the energy density in the alternative model in (34) can be obtained using Campbell's theorem [21]:

$$
\begin{aligned}
\mathrm{E}[\tilde{g}(X)] & =\gamma \lambda_{e} \int_{0}^{\infty} e^{-\frac{r^{2}}{\nu}} 2 \pi r d r \\
& =\pi \gamma \psi
\end{aligned}
$$

Hence, the expectation ratio $\mathrm{E}[\tilde{g}(X)] / \mathrm{E}[g(X)]=1+\pi \psi$. As $\psi$ is typically much smaller than 1 , the ratio is close to one.

Using the result and the technique in [32] for bounding the tail probability of a shot-noise process, it can be obtained that

$$
\begin{aligned}
\operatorname{Pr}(\tilde{g}(X)>x)= & \operatorname{Pr}(g(X)>x)+\operatorname{Pr}(g(X) \leq x) \times \\
& \operatorname{Pr}\left(\sum_{Y \in \Phi \cap \bar{B}\left(X, f^{-1}(x / \gamma)\right)} f(|X-Y|)>\frac{x}{\gamma}\right) \\
\leq & \operatorname{Pr}(g(X)>x)+\operatorname{Pr}(g(X) \leq x) \times \\
& \frac{\mathrm{E}\left[\sum_{Y \in \Phi \cap \bar{B}\left(X, f^{-1}(x / \gamma)\right)} f(|X-Y|)\right]}{x / \gamma} \\
= & \operatorname{Pr}(g(X)>x)+\operatorname{Pr}(g(X) \leq x) \times \pi \lambda_{e} \nu .
\end{aligned}
$$

The inequality can be rewritten as

$$
\begin{aligned}
\operatorname{Pr}(\tilde{g}(X)>x)-\operatorname{Pr}(g(X)>x) & \leq \pi \lambda_{e} \nu \times \operatorname{Pr}(g(X) \leq x) \\
& \leq \pi \lambda_{e} \nu \epsilon
\end{aligned}
$$

where the constant $\epsilon \in(0,1)$ is related to the maximum outage probability of the renewable powered network and is close to zero in the operating regime of interest. It follows that the tail probabilities for both energy field models are similar.

\section{B. Energy Field Model with Power Law Energy Decay and Its Effect on Network Coverage}

In the preceding sections, the outage probability is analyzed based on the energy field model characterized by the exponential energy decay function of distance in (4). In this section, the analysis is extended to the alternative power law function $f^{\prime}$ defined as

$$
f^{\prime}(d)=\left(1+\frac{d^{2}}{\nu}\right)^{-1}
$$

where $f^{\prime}(d) \propto d^{-2}$ if $d$ is large. Note that $f^{\prime}(d) \rightarrow 0$ as $d \rightarrow \infty$ and $f^{\prime}(d) \rightarrow 1$ as $d \rightarrow 0$, having the desirable properties for an energy decay function. It is assumed that the BSs adopt channel-independent transmission and that the channel coefficients follow a chi-squared distribution with parameter $\omega$. A similar procedure can be followed to obtain results for other cases, however the key insights are identical to those from the current analysis. Therefore, the details are omitted for brevity.

Recall that the outage probability can be decomposed as $p_{\text {out }}^{\text {id }}=p_{a}+p_{b}$ with $p_{b}$ and $p_{a}$ defined in (11) and (12). From their definitions, the change on the energy field model by modifying the energy decay function affects only $p_{a}$ but not $p_{b}$. The resultant $p_{a}$ is analyzed as follows. By a slight abuse of notation, let the distance function $r(\cdot)$ in (5) and the energy intensity function $g(\cdot)$ in (3) also denote their counterparts corresponding to $f^{\prime}(\cdot)$ in $(37)$. Then

$$
r(x)=f^{\prime-1}(x / \gamma)=\sqrt{\nu(\gamma / x-1)} .
$$


This results in the following distribution of the energy intensity $g(X)$ for an arbitrary location $X \in \mathbb{R}^{2}$ :

$$
\begin{aligned}
\operatorname{Pr}(g(X) \leq x) & =e^{-\pi \lambda_{e} r^{2}(x)} \\
& =e^{-\pi \psi\left(\frac{\gamma}{x}-1\right)} .
\end{aligned}
$$

Then from (11), $p_{a}$ can be upper bounded as

$$
\begin{aligned}
p_{a} & \leq \operatorname{Pr}\left(\frac{g(X) H_{0} R_{0}^{-\alpha}}{K_{0}} \leq \frac{\theta}{\eta}\right) \\
& =e^{\pi \psi} \mathrm{E}\left[\exp \left(-\frac{\pi \psi \gamma \eta H_{0}}{\theta K_{0} R_{0}^{\alpha}}\right)\right] \\
& =\left(\frac{\theta}{\pi \psi \eta \gamma}\right)^{\omega} e^{\pi \psi} \mathrm{E}\left[K_{0}^{\omega}\right] \mathrm{E}\left[R_{0}^{\alpha \omega}\right] \\
& =\left(\frac{\theta}{\pi \psi \eta \gamma}\right)^{\omega} e^{\pi \psi}\left(\frac{2}{3 \sqrt{3} \lambda_{b}}\right)^{\frac{\alpha \omega}{2}} \mathrm{E}\left[K_{0}^{\omega}\right]
\end{aligned}
$$

where $\mathrm{E}\left[K_{0}^{\omega}\right]$ is given in (19). Combining the last inequality with Lemmas 2 and 3 gives the main result of this section.

Proposition 7. Consider the scenario where BSs adopt channel-independent transmission and are powered by on-site harvesters. Suppose that the channel coefficients follow i.i.d. chi-squared distributions and that the energy density decay follows the function $f^{\prime}$ in (37), the outage probability satisfies

$$
p_{\text {out }}^{\text {id }} \leq\left(\frac{\theta}{\pi \psi \eta \gamma}\right)^{\omega} e^{\pi \psi}\left(\frac{2}{3 \sqrt{3} \lambda_{b}}\right)^{\frac{\alpha \omega}{2}} \mathrm{E}\left[K_{0}^{\omega}\right]+\frac{c_{3} \theta \lambda_{u} \mathrm{E}\left[H_{0}^{-1}\right]}{\lambda_{b}^{1+\frac{\alpha}{2}} \eta \gamma} .
$$

Moreover, as $\eta \gamma \rightarrow \infty$,

$p_{\text {out }}^{\text {id }} \preceq c_{5}\left[\left(\frac{1}{\pi \psi}\right)^{\omega} e^{\pi \psi}+\frac{1}{\Gamma(\omega+1)}\right](\eta \gamma)^{-\omega}+O\left((\eta \gamma)^{-\omega-1}\right)$

where the constant

$$
c_{5}=\mathrm{E}\left[K_{0}^{\omega}\right] \theta^{\omega}\left(\frac{2 \lambda_{b}}{3 \sqrt{3}}\right)^{\frac{\omega \alpha}{2}} .
$$

Recall that the energy field influences the outage probability via its spatial variation and maximum harvested power, determined by the parameters $\psi$ and $\eta \gamma$, respectively. The results show that the component of the outage probability due to energy randomness scales as $\psi^{-\omega}$ if $\psi$ is small. Moreover, as $\eta \gamma$ increases, the probability decreases following the power law $(\eta \gamma)^{-\omega}$ as observed from Proposition (7). The scaling law $(\eta \gamma)^{-1}$ is more gradual due to a loose bound resulting from the use of Markov inequality in the derivation.

Comparing the results in Proposition 7 with those in Proposition 2 corresponding to the exponential energy decay function, the outage probability for the current case is less sensitive to the changes on $\psi$ but more sensitive to those on $\eta \gamma$. The reason is that the power law energy decay function results in less spatial fluctuation in the energy field with respect to the double-exponential function.

\section{Simulation Results}

Unless otherwise specified, the simulation settings are as follows. The BSs and mobiles have densities of $\lambda_{b}=$ $0.78 / \mathrm{km}^{2}$ and $\lambda_{m}=7.8 / \mathrm{km}^{2}$, respectively, corresponding to an average cell radius of 500 meter and 10 users per cell. For

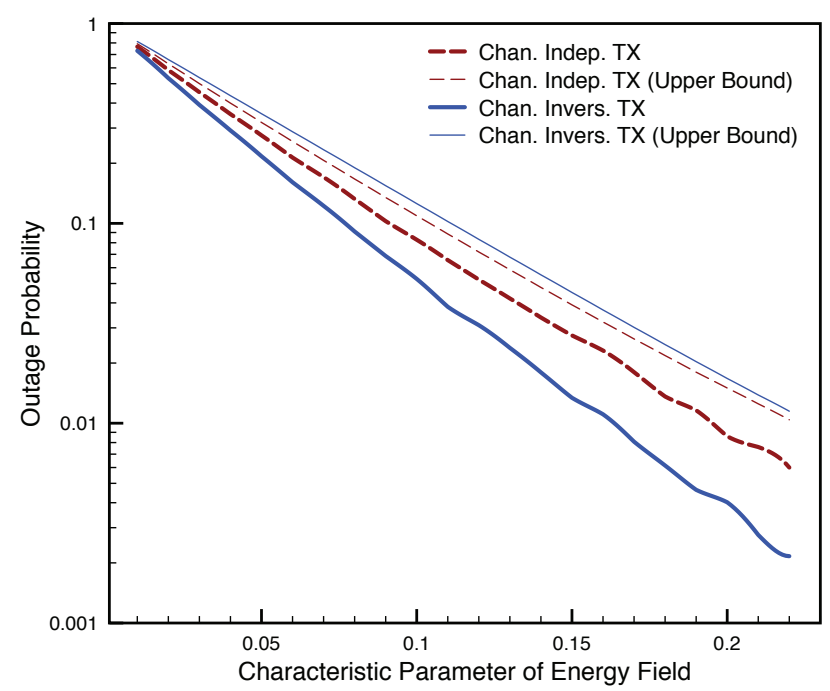

Fig. 4. Outage probability versus the characteristic parameter of the energy field for the scenario of on-site harvesters.

propagation, the reference path loss is $70 \mathrm{~dB}$ measured for a propagation distance of $100 \mathrm{~m}$ [33], the pathloss exponent $\alpha=4$, and the noise power is $-90 \mathrm{dBm}$. The product of the harvester aperture and the maximum energy intensity, $\gamma \eta$, gives the maximum power a harvester can generate, which is fixed as $\gamma \eta=1 \mathrm{~kW}$ for an on-site harvester and $10 \mathrm{~W}$ for a distributed one. For the case of distributed harvesters, the harvester density is $15.6 / \mathrm{km}^{2}$ that is twice of the BS density. The SNR threshold $\theta$ is 8 corresponding to a spectrum efficiency of about $3.2 \mathrm{bit} / \mathrm{s} / \mathrm{Hz}$. The fading coefficients are i.i.d. and distributed as $\max \left(|\mathcal{C N}(1,1)|^{2}, 0.1\right)$, where the $\mathcal{C N}(1,1)$ random variables model Rician fading and the truncation at 0.1 accounts for the avoidance of deep fading by scheduling.

Consider the scenario of on-site harvesters. The curves of outage probability versus the characteristic parameter $\psi$ are plotted in Fig. 4 for both the channel-independent and channel inversion transmissions. The upper bounds on the outage probabilities as given in Propositions 1 and 3 are also shown. The outage probabilities are observed to decay exponentially with increasing $\psi$ as predicted by analysis. Channel inversion transmission perform better than the other transmission scheme. The probability bound for channel inversion transmission is not as tight as that for channel-independent transmission as the former is based on the union bound [see (2)]. As the product $\gamma \eta$ is large, significant power can be harvested even at locations far from energy centers. Consequently, the outage probability is close to zero even for a small characteristic parameter (e.g., 0.2).

Next, consider the scenario of distributed harvesters where the characteristic parameter is fixed as 0.05. In Fig. 5, the outage probability is plotted against the number of harvesters connected to a single aggregator for energy aggregation (that is approximately equal to $\lambda_{h} / \lambda_{a}$ ). For comparison, the figure also shows the asymptotic upper bound on outage probabilities as given in Proposition 5 as well as those generated by simulation and replacing transmission powers with the asymptotic 


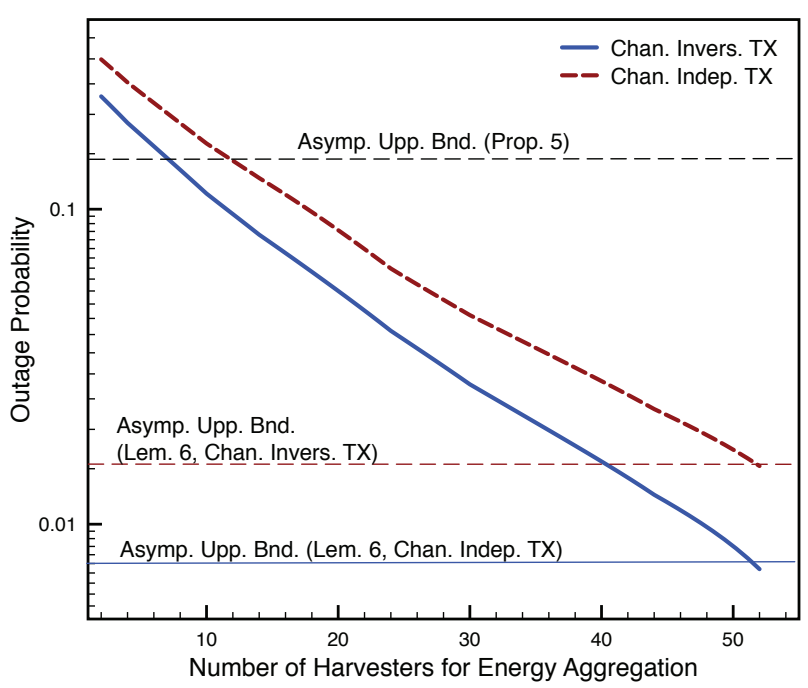

Fig. 5. Outage probability versus the number of harvesters for energy aggregation for the scenario of distributed harvesters. The characteristic parameter is fixed as $\psi=0.05$.

lower bound in Lemma 6. Aggregation loss is omitted assuming sufficiently high transmission voltage. It is observed that energy aggregation dramatically reduces outage probabilities, indicating energy randomness as the main reason for outage events. Most of the aggregation gain can be achieved with less than 50 harvesters per aggregator. For a large number of harvesters, the limits of outage probability depend only slightly on the randomness of the energy field and is mostly affected by channel fading as well as mobile random locations for the case of channel inversion transmission. Last, the asymptotic upper bound from Proposition 5 is observed to be loose due to the use of Markov's inequality but the other asymptotic bounds based on Lemma 6 are tight.

\section{CONCLUSION}

In this paper, a novel spatial model of renewable energy has been presented and applied to quantify the effect of energy spatial randomness on the coverage of cellular networks powered by energy harvesting. Moreover, the proposed technique of energy aggregation has demonstrated that new network architectures can be designed to cope with energy spatial variations. This work provides a useful analytical framework for designing large-scale energy harvesting networks. Moreover, it opens an interesting research direction of redesigning communication techniques e.g., multi-cell cooperation and resource allocation, as a means to achieve network reliability in the presence of energy randomness.

\section{APPENDIX}

\section{A. Proof of Lemma 1}

By substituting $P_{0}=\eta g\left(B_{0}\right)$ and (6) into (11),

$$
\begin{aligned}
p_{a}= & \left(\frac{\theta}{\gamma \eta}\right)^{\pi \psi} \mathrm{E}\left[\left(K_{0} H_{0}^{-1} R_{0}^{\alpha}\right)^{\pi \psi} \mid K_{0} H_{0}^{-1} R_{0}^{\alpha} \leq \eta \gamma\right] \times \\
& \operatorname{Pr}\left(K_{0} H_{0}^{-1} R_{0}^{\alpha} \leq \gamma\right) .
\end{aligned}
$$

It follows that

$$
p_{a} \leq\left(\frac{\theta}{\gamma \eta}\right)^{\pi \psi} \mathrm{E}\left[K_{0}^{\pi \psi}\right] \mathrm{E}\left[H_{0}^{-\pi \psi}\right] \mathrm{E}\left[R_{0}^{\alpha \pi \psi}\right] .
$$

By substituting $\bar{R}_{0}$ into (40),

$$
p_{a} \leq\left(\frac{\theta}{\gamma \eta \lambda_{b}^{\frac{\alpha}{2}}}\right)^{\pi \psi} \mathrm{E}\left[K^{\pi \psi}\right] \mathrm{E}\left[H_{0}^{-\pi \psi}\right] \mathrm{E}\left[\bar{R}_{0}^{\alpha \pi \psi}\right] .
$$

It follows from the inequality $\bar{R}_{0} \preceq D$ and the distribution of $D$ in (13) that

$$
\mathrm{E}\left[\bar{R}_{0}^{\alpha \pi \psi}\right] \leq \frac{2 c_{2}^{\pi \psi}}{2+\alpha \pi \psi}
$$

where $c_{2}$ is defined in the lemma statement. By combining the inequality with (41),

$$
p_{a} \leq \frac{2}{2+\alpha \pi \psi}\left(\frac{c_{2} \theta}{\gamma \eta \lambda_{b}^{\frac{\alpha}{2}}}\right)^{\pi \psi} \mathrm{E}\left[K_{0}^{\pi \psi}\right] \mathrm{E}\left[H_{0}^{-\pi \psi}\right] .
$$

If $\pi \psi \leq 1$, the upper bound on the outage probability can be reduced using Jensen's inequality as

$$
p_{a} \leq \frac{2}{2+\alpha \pi \psi}\left(\frac{c_{2} \theta \mathrm{E}\left[K_{0}\right] \mathrm{E}\left[H_{0}^{-1}\right]}{\gamma \eta \lambda_{b}^{\frac{\alpha}{2}}}\right)^{\pi \psi} .
$$

If $\pi \psi>1$, since larger $\psi$ leads to more harvested energy and thus smaller outage probability, the inequality in (43) holds by setting $\pi \psi=1$ :

$$
p_{a} \leq \frac{2 c_{2} \theta \mathrm{E}\left[K_{0}\right] \mathrm{E}\left[H_{0}^{-1}\right]}{(2+\alpha \pi \psi) \gamma \eta \lambda_{b}^{\frac{\alpha}{2}}} .
$$

Combining (44) and (45) and substituting $\mathrm{E}\left[K_{0}\right]=\lambda_{u} / \lambda_{b}$ gives the desired result.

\section{B. Proof of Lemma 2}

Using Markov's inequality, $p_{b}$ defined in (12) can be bounded as

$$
\begin{aligned}
p_{b} & \leq \frac{\theta \mathrm{E}\left[K_{0} H_{0}^{-1} R_{0}^{\alpha}\right]}{\eta \gamma} \\
& =\frac{\theta \mathrm{E}\left[K_{0}\right] \mathrm{E}\left[H_{0}^{-1}\right] \mathrm{E}\left[R_{0}^{\alpha}\right]}{\eta \gamma} \\
& =\frac{\theta \lambda_{u} \mathrm{E}\left[H_{0}^{-1}\right] \mathrm{E}\left[R_{0}^{\alpha}\right]}{\eta \gamma \lambda_{b}}
\end{aligned}
$$

where the equality in (46) holds since $K_{0}, H_{0}$ and $R_{0}$ are independent and (47) follows by substituting $\mathrm{E}\left[K_{0}\right]=\lambda_{u} / \lambda_{b}$. Using the definition of $\bar{R}_{0}$ and the inequality in (13), it is obtained that

$$
\mathrm{E}\left[R_{0}^{\alpha}\right] \leq\left(\frac{2}{3 \sqrt{3} \lambda_{b}}\right)^{\frac{\alpha}{2}}
$$

Substituting (48) into (47) gives the desired result. 


\section{Proof of Lemma 3}

The probability $p_{b}$ in (12) can be bounded as

$$
\begin{aligned}
p_{b} & =\operatorname{Pr}\left(K_{0} H_{0}^{-1} \bar{R}_{0}^{\alpha}>\frac{\eta \gamma}{\lambda_{b}^{\frac{\alpha}{2}} \theta}\right) \\
& \leq \operatorname{Pr}\left(K_{0} H_{0}^{-1} D^{\alpha}>\frac{\eta \gamma}{\lambda_{b}^{\frac{\alpha}{2}} \theta}\right) \\
& \leq \operatorname{Pr}\left(K_{0} H_{0}^{-1}>\frac{\eta \gamma}{\left(\frac{2 \lambda_{b}}{3 \sqrt{3}}\right)^{\frac{\alpha}{2}} \theta}\right) .
\end{aligned}
$$

Thus, analyzing the scaling law of $p_{b}$ as the maximum harvested power $\eta \gamma$ increases is equivalent to characterizing the large deviation of the product of the two RVs $K_{0}$ and $H_{0}^{-1}$. This relies on Breiman's Theorem stated as follows [28, Corollary 3.6].

Lemma 7 (Breiman's Theorem). Suppose that $X$ and $Y$ are two independent non-negative $R V$ s where $X$ is a regularly varying $R V$ with the exponent $\omega>0$ and $\mathrm{E}\left[Y^{\omega+\epsilon}\right]<\infty$ for some $\epsilon>0$. Then

$$
\operatorname{Pr}(X Y>t) \sim \mathrm{E}\left[Y^{\omega}\right] \operatorname{Pr}(X>t), \quad t \rightarrow \infty .
$$

It is known that the moment of the Poisson RV $K_{0}$ exists as given below. Thus, $\mathrm{E}\left[K_{0}^{\omega+\epsilon}\right]<\infty$ for some $\epsilon>0$. Given this condition and the assumption of $H_{0}^{-1}$ being a regularly varying $\mathrm{RV}$, the first result in the lemma statement can be obtained from (18), (49) and Lemma 7. The second result follows by substituting the distribution of $H_{0}$ in (18).

\section{REFERENCES}

[1] K. A. Adamson and C. Wheelock, "Off-grid power for mobile base stations (report)," report, Navigant Research, 2013.

[2] K. Huang, "Spatial throughput of mobile ad hoc networks with energy harvesting," IEEE Trans. on Information Theory, vol. 59, pp. 7597-7612, Nov. 2013.

[3] H. S. Dhillon, Y. Li, P. Nuggehalli, Z. Pi, and J. G. Andrews, "Fundamentals of heterogeneous cellular networks," IEEE Trans. on Wireless Comm., vol. 13, pp. 2782-2797, May 2014.

[4] S. Lee, R. Zhang, and K. Huang, "Opportunistic wireless energy harvesting in cognitive radio networks," IEEE Trans. on Wireless Comm., vol. 12, pp. 4788-4799, Sep. 2013.

[5] K. Huang and V. K. N. Lau, "Enabling wireless power transfer in cellular networks: Architecture, modelling and deployment," IEEE Trans. on Wireless Comm., vol. 13, pp. 902-912, Feb. 2014.

[6] M. Haenggi, J. G. Andrews, F. Baccelli, O. Dousse, and M. Franceschetti, "Stochastic geometry and random graphs for the analysis and design of wireless networks," IEEE Journal on Selected Areas in Comm., vol. 27, pp. 1029-1046, Jul. 2009.

[7] P. Gupta and P. R. Kumar, "The capacity of wireless networks," IEEE Trans. on Information Theory, vol. 46, pp. 388-404, Feb. 2000.

[8] S. Boyd, A. Ghosh, B. Prabhakar, and D. Shah, "Randomized gossip algorithms," IEEE Trans. on Information Theory, vol. 52, pp. 25082530, Jun. 2006.

[9] N. A. C. Cressie, Statistics for Spatial Data. John Wiley \& Sons, 1993.

[10] J. P. Chiles and P. Delfiner, Geostatistics: Modeling spatial uncertainty. John Wiley and Sons, 2nd ed., 2012.

[11] O. Ozel and S.Ulukus, "Information-theoretic analysis of an energy harvesting communication system," in Proc. of IEEE Personal, Indoor and Mobile Radio Comm., Sep. 26-29 2010.

[12] C. Ho and R. Zhang, "Optimal energy allocation for wireless communications with energy harvesting constraints," IEEE Trans. on Signal Processing, vol. 60, pp. 4808-4818, Sep. 2012.
[13] O. Ozel, K. Tutuncuoglu, J. Yang, S. Ulukus, and A. Yener, "Transmission with energy harvesting nodes in fading wireless channels: Optimal policies," IEEE Journal on Selected Areas in Comm., vol. 29, pp. 17321743, Aug. 2011.

[14] K. Tutuncuoglu and A. Yener, "Sum-rate optimal power policies for energy harvesting transmitters in an interference channel," Journal of Comm. and Networks, vol. 14, pp. 151-161, Feb. 2012.

[15] C. Huang, R. Zhang, and S. Cui, "Throughput maximization for the gaussian relay channel with energy harvesting constraints," IEEE Journal on Sel. Areas in Comm. Areas in Comm., vol. 31, pp. 1469-1479, Aug. 2013.

[16] F. Iannello, O. Simeone, and U. Spagnolini, "Medium access control protocols for wireless sensor networks with energy harvesting," IEEE Trans. on Comm., vol. 60, pp. 1381-1389, May 2012.

[17] B. Devillers and D. Gunduz, "A general framework for the optimization of energy harvesting communication systems with battery imperfections," Journal of Comm. and Networks, vol. 14, pp. 130-139, Feb. 2012.

[18] Renewable Resource Data Center, National REnewable Energy Lab., USA. (Web: http://www.nrel.gov/rredc/)

[19] Z. Sen and A. D. Sahin, "Spatial interpolation and estimation of solar irradiation by cumulative semivariograms," Solar Energy, vol. 71, pp. 11-21, Jan. 2001.

[20] W. R. Goodin, G. J. McRae, and J. H. Seinfeld, "A comparison of interpolation methods for sparse data: application to wind and concentration fields," Journal of Applied Meteorology, vol. 18, pp. 761-771, Jun. 1979.

[21] J. F. C. Kingman, Poisson processes. Oxford University Press, 1993.

[22] S. L. Barnes, "A technique for maximizing details in numerical weather map analysis," Journal of Applied Meteorology, vol. 3, pp. 396-409, Apr. 1964

[23] Z. Sen, "Solar energy in progress and future research trends," Progress in Energy and Combustion Science, vol. 30, pp. 367-416, Apr. 2004.

[24] J. G. Andrews, F. Baccelli, and R. K. Ganti, "A tractable approach to coverage and rate in cellular networks," IEEE Trans. on Comm., vol. 59, pp. 3122-3134, Nov. 2011.

[25] Y.-K. Chia, S. Sun, and R. Zhang, "Energy cooperation in cellular networks with renewable powered base stations," IEEE Trans. on Wireless Comm., vol. 13, pp. 6996-7010, Dec. 2014.

[26] Y. Guo, J. Xu, L. Duan, and R. Zhang, "Joint energy and spectrum cooperation for cellular communication systems," IEEE Trans. on Comm., vol. 62, pp. 3678-3691, Oct. 2014.

[27] J. Serra, "Boolean random functions," Journal of Microscopy, vol. 156, pp. 41-63, Oct. 1989

[28] D. B. H. Cline and G. Samorodnitsky, "Subexponentiality of the proudct of independent random variables," Stochastic Processes and their Applications, vol. 49, pp. 75-98, Jan. 1994

[29] D. Denisov, S. Foss, and D. Korshunov, "On lower limits and equivalences for distribution tails of randomly stopped sums," Bernoulli, vol. 14, pp. 391-404, May 2008.

[30] M. Mitzenmacher and E. Upfal, Probability and Computing. Cambridge University Press, 2005.

[31] S. B. Lowen and M. C. Teich, "Power-law shot noise," IEEE Trans. on Information Theory, vol. 36, pp. 1302-1318, Jun. 1990.

[32] S. P. Weber, J. G. Andrews, X. Yang, and G. de Veciana, "Transmission capacity of wireless ad hoc networks with successive interference cancelation," IEEE Trans. on Information Theory, vol. 53, pp. 27992814, Aug. 2007.

[33] T. S. Rappaport, Wireless Communications Principles and Practice. Upper Saddle River, NJ: Prentice Hall, second ed., 2002. 